\title{
Natural selection in chemical evolution
}

\author{
Chrisantha Fernando ${ }^{a, b, *}$, Jonathan Rowe ${ }^{a}$ \\ ${ }^{\mathrm{a}}$ School of Computer Science, University of Birmingham, B15 2TT, UK \\ ${ }^{\mathrm{b}}$ Collegium Budapest (Institute for Advanced Study), 2 Szentharomsag utca, H-1014 Budapest, Hungary
}

Received 11 October 2006; received in revised form 30 January 2007; accepted 31 January 2007

Available online 12 February 2007

\begin{abstract}
We propose that chemical evolution can take place by natural selection if a geophysical process is capable of heterotrophic formation of liposomes that grow at some base rate, divide by external agitation, and are subject to stochastic chemical avalanches, in the absence of nucleotides or any monomers capable of modular heredity. We model this process using a simple hill-climbing algorithm, and an artificial chemistry that is unique in exhibiting conservation of mass and energy in an open thermodynamic system. Selection at the liposome level results in the stabilization of rarely occurring molecular autocatalysts that either catalyse or are consumed in reactions that confer liposome level fitness; typically they contribute in parallel to an increasingly conserved intermediary metabolism. Loss of competing autocatalysts can sometimes be adaptive. Steady-state energy flux by the individual increases due to the energetic demands of growth, but also of memory, i.e. maintaining variations in the chemical network. Self-organizing principles such as those proposed by Kauffman, Fontana, and Morowitz have been hypothesized as an ordering principle in chemical evolution, rather than chemical evolution by natural selection. We reject those notions as either logically flawed or at best insufficient in the absence of natural selection. Finally, a finite population model without elitism shows the practical evolutionary constraints for achieving chemical evolution by natural selection in the lab.
\end{abstract}

(C) 2007 Elsevier Ltd. All rights reserved.

Keywords: Origin of metabolism; Autocatalytic cycle; Liposome; Natural selection; Chemical evolution

\section{Introduction}

Chemical evolution is the synthetic process by which the complex organic molecules that constituted the first units of life were formed. The prototypical example has been Miller's experiment (Miller, 1953) in which non-random molecules, e.g. formic acid, glycine, glycolic acid, alanine, etc. are produced (Miller and Orgel, 1974, p. 85). Ultimately however, such setups produce a combinatorial explosion of polymers (Schuster, 2000) that are not typically subject to natural selection.

What modifications must be made to this type of experiment to allow at least one of the following outcomes: 'open-ended evolution' (Bedau et al., 2000); the origin of

\footnotetext{
${ }^{*}$ Corresponding author. School of Computer Science, University of Birmingham, B15 2TT, UK. Tel.: + 44121 2360140; fax: + 7905527532.

E-mail addresses: c.t.fernando@cs.bham.ac.uk (C. Fernando), J.E.Rowe@cs.bham.ac.uk (J. Rowe).
}

basic autonomy, i.e. a dissipative system capable of the recursive generation of functional constraints (Ruiz-Mirazo et al., 2004); a process ultimately capable of the production of nucleic acids or other modular replicators with unlimited heredity potential (Maynard-Smith and Szathmary, 1995; Szathmary, 2000); identification of "the course of evolution by which the determinate order of biological metabolism developed out of the chaos of intercrossing reactions" (Oparin, 1964); the coupled cycling of bioelements (Morowitz, 1968, 1971); the maximization of entropy production by a biosphere (Kleidon, 2004); the minimal unit of life (Ganti, 2003a, b); or an autopoetic unit (Maturana and Verela, 1992)?

The process producing increasingly organized chemical supersystems is prebiotic evolution (Ganti, 2003b, p. 311; Hogeweg and Takeuchi, 2003). Prebiotic evolutionists divide into two schools: the replicator first (Haldane, 1954; Miller and Orgel, 1974; Eigen and Schuster, 1977) and the metabolism first (Oparin, 1924; Fox and Dose, 
1977; Dyson, 1985; Morowitz, 1992). The metabolism first group claim that organizing principles must have acted prior to hereditary variation by micro-mutation, i.e. prior to template replication. However, increasingly there is an admission that "the suggestion that relatively pure, complex organic molecules might be made available in large amounts via a self-organizing, autocatalytic cycle might, in principle, help to explain the origin of the component monomers" necessary for modular replicators (Orgel, 2000). If metabolism is the set of chemical reactions producing the constituents of the organism, it presupposes a physically distinct individual. Chemical organization theory (Dittrich and Speroni, 2007) is insufficient to define organism metabolism; there is no instance of an organism metabolism that is a closed and self-maintaining set, i.e. waste products are produced, and recycled outside but not inside the organism. Metabolism is also simultaneously more than a closed and self-maintaining set since there is exponential growth, and eventually the material is transformed into a physically distinct offspring. Therefore a consideration of metabolism first approach entails boundaries first or individuals first.

We assume that an important mechanism of variation in 'prebiotic evolution', of the metabolism first kind, was initially identical to the mechanism of variation in chemical evolution; i.e. stochastic reaction avalanches (Wachtershauser, 1992). The model presented here is heuristic (Maynard-Smith, 1986) and proposes that chemical evolution by natural selection is the mechanism responsible for prebiotic evolution, in contrast to the following theories.

Based on Eigen's proposal in 1971 of a reflexively autocatalytic protein set (Eigen, 1971), Stuart Kauffman extended Freeman Dyson's idea of a phase transition resulting in the spontaneous formation of such sets (Kauffman, 1986; Dyson, 1985). Kauffman's mechanism assumes: (i) a large set of abundant food molecules from which members of the set are produced; (ii) molecules have a certain probability of catalysing the production of other molecules; and (iii) each species in the set is reflexively autocatalytic (Farmer et al., 1986; Bagley and Farmer, 1991). These are not the conditions at the end of the Miller experiment, which although containing polymers of amino acids, does not seem to contain polymer replicators in high abundance. Kauffman's model assumes a significant extent of self-organization to produce the high numbers of successful autocatalysts capable of co-existence in the same reactor, when this is in fact what we must explain. Irrespective of the probability of catalysis or autocatalysis (Jain and Krishna, 1998; Mossel and Steel, 2005) Kauffman ignores the problem of side-reactions (Szathmary, 2000). He does not consider that as well as a probability of catalysed addition of a species to the set, there is an even greater probability of the catalysed removal of a species from the set, if such catalysts are indeed randomly generated. Even by using encoded molecules (Lee et al., 1997), it has not been possible to produce a reflexive autocatalytic set (Szathmary et al., 2005). Thus, Kauffman has proposed a self-organizing principle distinct from natural selection that does not work if we take the idea of side-reactions seriously; moreover, he assumes a priori a massive diversity of co-existing autocatalytic replicators, which is one of the things we must explain.

Another model of chemical evolution is Walter Fontana and Leo Buss' $\lambda$-calculus, in which they claim "organizations arise in a system lacking any formulation of Darwinian selection" (Fontana and Buss, 1994, p. 1). In their model, the absence of conservation of mass or energy brings into serious doubt whether their findings could be replicated in a physical well-mixed chemical flow reactor. $\lambda$ objects collide and react in a flow reactor. Random removal of $\lambda$-objects is a selective pressure at the level of $\lambda$-objects, so if they become capable of multiplication and variation they will be subject to natural selection. This is observed in the formation of L0 organizations consisting of self-copiers of form $\lambda x . x$, which form unstable hypercycles. When the authors introduce the assumption that singlestep copy operations are disallowed, L1 organizations form consisting of sets of self-reproducing $\lambda$-objects, which are stable against the introduction of L0 self-copiers at a later stage. They claim that no natural selection could have taken place between L1 organizations, so arguing for a selforganizing principle that could establish increasingly complex 'self-reproducing' metabolisms in the absence of natural selection at the level of the L1 organization. Eors Szathmary has correctly pointed out that it is not necessarily the case that only one L1 organization can exist in the flow-reactor, and therefore that natural selection could in fact take place (Szathmary, 1995). Even single constituents, if lost, may compromise an entire L1 organization. In any case, it is not clear whether even natural selection between multiple L1 organizations would be sufficient in a well-mixed flow reactor to allow continuing chemical complexification. Decker maintained a formose cycle in a flow reactor, but found no tendency for continued complexification, as Fontana and Buss' model predicts (Decker et al., 1982). One reason is the production of tapping (polymeric) side-products that are not themselves autocatalytic, but which increase the food threshold of the autocatalytic constituents (Decker et al., 1982; King, 1982). These are problems peculiar to systems with conservation of matter. In any case, in the world of $\lambda$ calculus, limiting the maximum length of expressions has artificially prevented the equivalent of such tapping by elongation reactions.

Another self-organizing principle has been put forward by Harold Morowitz who argues that life is 'driven by radiant energy' to attain complexity in the form of coupled cycling of material (Morowitz, 1992, p. 121). ${ }^{1}$ Although Morowitz is careful to point out that "complexity alone is an insufficient measure for characterizing the transition

\footnotetext{
${ }^{1}$ Morowitz's argument for energy driven self-organization is not intended to be specific to radiant energy but for any high quality energy source.
} 
from nonliving to living" (Morowitz, 1992, p. 123) he does go on to claim that the Miller type experiments "indicate the great potential of a directed energy input to organize a system". Organization being defined as compressible complexity (Morowitz, 1992, p. 127; McGregor and Fernando, 2005). The continued steady state flux through a Benard cell or a cloud does not arise because the physical properties of the Benard cell or the cloud were ordered by the heat flux itself. Rather, it is the response of a material that makes up these systems to the laws of convection, inertia, and gravity that serendipitously permit these particular systems to maintain a steady-state heat flux. No-one can deny that cycles of matter and energy occur in the biosphere, and individual organisms, but they are not characteristic of life since abiotic cycles exist. No-one can deny either that "steady-state organic reaction networks maintained away from equilibrium by the input of electronic excitation will be characterised by flows of matter around closed loops in the network." (, Morowitz, 1992, p. 117). However, we can deny that it is the case that all systems that are driven by the supply of external energy become organized; most will lose the capacity for recycling, by the formation of enlarging and non-recyclable sinks. Morowitz does not explain the mechanism by which only a small subset of systems that are driven by external energy increase their rate of recycling. We propose that natural selection is the principle means for producing the increasingly complex cyclic systems capable of increased steady state energy flux, and that vice versa, an initial steady state energy flux is necessary to sustain the first spontaneously formed natural selection machine. Thus, we do not agree with Morowitz' hypothesis that energy flux is a driving force for organization, but believe steady state energy flux is necessary for the maintenance of a 'natural selection machine'.

Recently, Dewer, who seeks to give a thermodynamic explanation for MaxEP (Dewer, 2005), has made the claim that systems with sufficient degrees of freedom, driven by an external energy source, maximize entropy production as an inevitable statistical consequence, and that this is a force for order. However, so far Dewer's model has been shown to apply to a very limited set of cases, e.g. convection systems, and his theory is incapable of explaining selforganization in chemical systems in which side-reactions can increase the size of the sink. In the later kind of system, our model lends support to the hypothesis that natural selection explains MaxEP.

\section{Chemical evolution by natural selection}

Natural selection is an algorithmic process that occurs in populations of entities capable of multiplication, variation and heredity (Maynard-Smith, 1986). What is the simplest 'machine' capable of sustaining natural selection, that was the most likely to form spontaneously? Oparin proposed natural selection between spontaneously formed dissipative coazervates, structures composed of initially randomly aggregated polypeptides (Oparin, 1938, p. 190). ${ }^{2}$ In an early presentation, Oparin distinguished the coazervates made in the lab (artificial coazervates) from coazervates that may have formed naturally, the later being capable of internal reactions and rare chemical transformations, with selection between coazervates favouring those capable of absorbing material from the outside medium more strongly (Oparin, 1938). Later, Oparin suggested that polypeptide composition would be the substrate for variation (Oparin, 1964 , p. 70). No replicating coazervates were demonstrated. Sidney Fox experimented with microspheres composed of polypeptides, working along essentially the same principle whereby division depends on self-replication of the constituents, or at least the non-random polycondensation of amino acids. Budding occurred, but with undetermined heredity (Fox and Dose, 1977, p. 248). In another variant of this theme, Folsome observed that the "thin oily scum" on the water surface of Miller type experiments formed microstructures that she claimed grew exponentially and then sank to the bottom of the flask (Folsome, 1979). In all cases the mechanism of natural selection is the aggregation if partly hydrophobic constituents in aqueous systems. Folsome proposed that the first microstructures grow by light driven heterotrophic anhydrous polycondensation of amino acids driven by pyrophosphates' conversion to phosphate; pyrophosphate being produced in 'photosynthesis by the primary metabolism of protocells' (Folsome, 1979, p. 110). Such an ordered system could not form spontaneously, and exists only in theory. Krishna Bahadur in India, working at the same time as Fox, showed that formaldehyde, ammonium phosphate, mineral salts and ammonium molybdate, when exposed to sunlight, formed spherical microstructures called "Jeewanu", (Ganti, 2003b, p. 329) (Bahadur, 1954; Bahadur and Ranganayaki, 1983) with catalytic activates. Arther Weber recently described a similar synthesis of proliferating microspherules from sugers and ammonia (Weber, 2005). Almost a century of research shows there are many ways to produce phase-separated individuals.

It is important to distinguish the mechanism of liposome growth and variation proposed here, from the idea of 'composome' based heredity in the Lipid World model

\footnotetext{
2"'In this way a natural selection of coazervates originated in its most primitive and simplest form, only the dynamically most stable colloidal systems securing for themselves the possibility of continued existence and evolution... With the background of 'natural selection' and by its strict control, further modifications must have proceeded along definite lines. In natural coazervate droplets, contrary to what happens in artificial coazervates, inorganic catalysts, such as hydrogen ions, iron oxide, etc., undoubtedly assume an important part, accelerating the definite chemical changes within the coazervate... Sooner or later [the coazervate] would have to break up into separate droplets or fragments simply under the influence of external mechanical forces, or this could result from the action of surface tension forces. Incidentally such fragmentation would be advantageous from the point of view of further growth ... since it would establish a more favourable relation between surface and mass and thus increase the absorption of dissolved substances." pp. 189-195. The Origin of Life. A.I. Oparin, 1938. (1953 Edition, Dover Publications).
} 
(Segre et al., 2000, 2001), which is akin to a population of reflexively autocatalytic sets isolated in compartments, and which is not capable of stable heredity. We propose that chemical avalanches produce autocatalysts that do not necessarily contribute to the growth of the liposome by catalysing the production of other autocatalysts in a reflexive manner, but by contributing to a common intermediary metabolism. The network diagrams in the results section reveal the evolution of a completely different organization to the 'composome' model.

Inspired principally by the experimental work of Oparin, Fox and Dose, Folsome, etc., we favour the hypothesis that the natural selection machine capable of forming the first cells was a geophysical process capable of spontaneously forming phase separated 'individuals', e.g. liposomes, undergoing division by externally imposed agitation, i.e. replication rather than self-replication. This may take the form of turbulent flows, shaking, convection currents in hydrothermal vents etc. In addition to the reliable sustenance of a reactor in which liposomes can grow and multiply, a source of internal liposome variation would have been required. Guenter Wachtershauser has considered in some detail the capacity for pre-template variation in a more complex surface bound 3 phase 'semi-cell' (Wachtershauser, 1992). Wachtershauser writes "Abandoning determinism (Popper, 1972, 1974, 1983c, 1990a) we come to see that chemical reality covers more than high-propensity ("deterministic") reactions. In fact, low-propensity ignitions of autocatalytic cycles (with highpropensity propagations once they have been ignited) seems to be the very chemical stuff of biochemical evolution." (Wachtershauser, 1992, p. 89). We model precisely such a process, referring to it as "variation by chemical avalanches"3. Szathmary classifies this process as holistic or attractor based heredity (Szathmary, 2000).

\section{A model of chemical evolution by natural selection}

The six assumptions we make are offered below.

Assumption 1. A reactor ('natural selection machine') capable of sustaining replicating phase-separated compartments arises spontaneously. Heterotrophically replicating

\footnotetext{
${ }^{3}$ Wachtershauser makes a distinction between hetero-origin theories which assume "prebiotic chemical reactions up to a complexity of, say, activated nucleotides between a multitude of theatres with diverse chemical conditions" (Wachtershauser, 1992, p 89) and his chemoautotrophic hypothesis. The model presented here assumes the intermediate position, with the "limited" heterotrophic origin of liposomes. We assume the geophysical reactor only being capable of the minimal number of synthesis reactions capable of producing replicating liposomes that can then undergo intra-liposomal avalanche type variation. We have no objection to the idea of surface-bounded hydrophobic compartments, rather than liposomes in aqueous solution, however, our model differs from Wachtershauser's because we assume that such compartments form by a limited heterotrophic process, not initially by an internal chemical autotrophic processes, which we assume would be possible but unlikely to occur with sufficient probability in the absence of a sufficiently rich set of high energy 'virgin' food materials.
}

liposomes exist, probably on a surface as in Wachtershauser's hypothesis of the Iron-Sulpher world. However, the dynamics considered here assume simply that replication of some phase separated individual, e.g. a liposome, is possible due to the incorporation of external food matter that results in proliferation. The crucial difference between this model and that of Wachtershauser's is that we assume that no specific intra-liposome chemical reactions are necessary for a base rate of liposome replication. Such a robust geophysical process of generating "virgin" liposomes and proliferating existing liposomes by incorporation of 'food molecules' is central to this hypothesis. We assume that some geochemical cycle is capable of spontaneously arising that continues the supply of 'food material' (the food set) to a reactor containing such liopsomes. Further, we assume that these liposomes divide due to external agitation. Arguably, this should be called replication not self-replication, nevertheless it is multiplication we need for natural selection (Maynard-Smith, 1986).

Assumption 2. 'Random' rare novel species are produced by rare reactions within the liposome. Novel rare reactions arise (either in the liposome or in the surrounding water phase) that may result in the production of a novel chemical species at extremely low copy number.

Assumption 3. There is an avalanche of effects due to a novel Chemical species. Some novel chemical species may undergo reactions that occur at sufficiently high rates that they influence the dynamics of a liposome within the average basal doubling period of the liposome. Of-course, by reasoning alone one can appreciate that a very rarely produced novel species present at very low copy number can only have a significant effect if it is autocatalytic since otherwise its copy number will not increase beyond one, and it will be lost by segregation instability. Most novel rare species will not be autocatalytic. By autocatalytic we mean that it grows exponentially, not that it is directly autocatalytic in one chemical step, for example it may be the case that the two 'copies' of the species are produced at two distant points in the reaction network. Furthermore, by reasoning alone one appreciates that even if the novel species is autocatalytic, other reactions in which it participates may not be of benefit to the liposome. A novel species has a certain probability of being in the lipid phase (so remaining within the liposome), and a certain probability of being in the water phase, so being extruded from the liposome. These are precisely the kind of sidereactions that have been ignored in Kauffman's models. Side-reactions occur as avalanches that randomly explore the adjacent possible chemical space. These avalanches consist of additions to the stoichiometic matrix, and are not restricted to catalysis, although catalysis may emerge due to the generation of cycles of production. Alternatively, avalanches may 'poison' catalytic cycles, again this is an emergent process in our model. Typically, an avalanche, even if it produces an autocatalytic topology 
will not be capable of sustaining that autocatalyst since the decay rate of the novel autocatalyst may be too high due to side-reactions also produced in the avalanche. This is the mechanism of chemical variation that contributes to variance in liposome fitness.

Assumption 4. Liposome fitness is proportional to the rate of production of a subset of lipophilic molecules (the growth set). The fitness of the liposome is a complicated function of its composition. However, for purposes of modelling we assume that liposome growth is proportional to the rate of production of some subset of lipid phase species by the reactions occurring between the liposome constituents and the externally supplied food set. We assume an artificial selection type experiment in which a batch reactor (envisioned to be a microfluidic reactor) is initialized with a supply of food molecules and a divided liposome from a parental reactor. For computational simplicity we will only model one liposome rather than a population of liposomes. The fitness is the extent of growth by the liposome within the batch reactor initialized with a food bolus at the start.

Assumption 5. Inheritance of liposome constituents occurs at division. During division which we impose at fixed time periods due to external agitation, approximately half of the lipophilic constituents of the liposome are inherited by each daughter cell. The water phase constituents, not being so inherited, are assumed to have been washed away in the water phase in which liposomes exist (A. Moreno, personal communication).

Assumption 6. A potential autotrophic reaction exists that is capable of utilizing light energy to drive an otherwise non-spontaneous reaction. There is conservation of mass and energy in chemistry. This imposes important constraints that define the properties of reaction networks. All chemical species have a defined free energy of formation, and material composition. The food set has a defined energy, as does the growth set. The autotrophic reaction may be 'discovered', i.e. if the reactants become synthesized due to a beneficial avalanche. The avalanche may be 'fixed' if it contributes to the growth of the liposome utilizing that reaction.

\section{Methods}

The above assumptions are modelled using a computer program. Simulated liposomes are selected that are capable of supra-basal growth rates due to the production of a predefined set of growth molecules. A hill-climbing algorithm (Rich and Knight, 1991) is used to assess the efficiency with which natural selection can generate 'adaptations' ${ }^{4}$ under the above variation and selection

\footnotetext{
${ }^{4} \mathrm{An}$ adaptation in this case is a chemical avalanche that increases the growth rate of the offspring that inherits the constituents that remain at the end of the life of the parent in which the avalanche originally occurred. It is a heritable variation that increases fitness. It is not sufficient to be an adaptation that an avalanche increases the fitness of the parent but results
}

constraints. This hill-climbing algorithm works by testing the fitness of a parent, and generating offspring from that parent until an offspring is produced whose fitness is greater than the fitness of the parent, in which case the offspring replaces the parent, and the algorithm iterates. The frequency of adaptation is the number of offspring whose fitness is greater than the parent divided by the number of offspring produced whose fitness is less than the parent.

\subsection{Overview of the algorithm}

Algorithm 1. An overview

Require: generation $=0 . t=0$. Initialize reactor with food set and initial reactions. Assume initial fitness of 'virgin' liposome $=0$. Generation_time $=10,000 \times 0.0001 \mathrm{~s}$. Timestep $=0.0001 \mathrm{~s}$.

1: for generation $=0$ to Max_Gen do

2: Create offspring reactor by taking $50 \%$ of liposome phase material present at end of parental generation, and replenishing the food set to its original concentration. 3: Generate Avalanche: Randomly (but according to thermodynamic and mass conservation constraints described later) create novel rare reactions and the subsequent high flux reaction avalanche. Initialize each novel species at very low concentration (e.g. $10^{-7} \mathrm{mM}$ ). 4: Simulate Offspring: Using the novel reaction network and initial concentrations use Eular Integration to simulate generation_time seconds of reaction dynamics, measuring fitness at 1000 time-step intervals.

5: Simulate 3 generations starting from the original offspring (with no chemical avalanches between divisions), in order to exclude non-inheritable avalanches

"adaptations". Store the fitness only of the 3rd offspring. 6: if 3rd Offspring fitness $>$ Parent fitness $\times 1.1$ (i.e. offspring fitness must be at least $10 \%$ greater than parent) 7: Replace Parental reactor with Offspring reactor. 8: end if 9: end for

\subsection{The artificial chemistry and initialization}

The purpose of the artificial chemistry is to model conservation of energy and mass. Conservation of mass is modelled as a constraint on the total number of 'atoms', i.e. characters in the strings that constitute a molecule.

\section{(footnote continued)}

in offspring that have no higher fitness than before. This would better be described as a non-transmissible developmental process. This can happen because the parental avalanche may give the parent a high fitness, but may result in a harmful bolus of material being transmitted to the offspring. However, it is expected to be the case that some avalanches that benefit the parent will also be able to benefit the offspring. 
Energy conservation is modelled by ensuring that all reaction paths from one molecule to another molecule have equal differences in free energy.

The artificial chemistry consists of 'molecules' which are $1 \mathrm{D}$ strings composed of the following 'atomic' units ' $a, b, c$ and $d^{\prime}$. Reactions consist exclusively of reversible and irreversible bimolecular rearrangements of the form

$A+B \Leftrightarrow C+D$,

$A+B \Rightarrow C+D$,

in which $C+D$ are random rearrangements of the letters in $A$ and $B$, for example

$a a b b+a b a \Leftrightarrow b b a a a+a b$.

The irreversible reactions are of two types; a light absorbing reaction, which is the only reaction defined at the outset...

$a b b+a b b \Rightarrow b a+a b b b$

and novel irreversible reactions, the need for which is described later. Each species has a defined free energy of formation $G_{f}$ that is either assigned at initialization or generated when a new reaction is produced. All novel reversible reactions are constrained to be spontaneous, that is, $\Delta G=G_{\text {products }}-G_{\text {reactants }}<0$. The equilibrium $K$ of a reversible reaction is defined as $K=\mathrm{e}^{-\Delta G / R T}$, where $R$ is the gas constant $\left(8.3144 \mathrm{~J} \mathrm{~mol}^{-1} \mathrm{~K}^{-1}\right)$ and $T$ is the temperature in Kelvin. Since thermodynamics underdetermines kinetics, we assume for simplicity that $k_{b}=0.01$ and $k_{f}=0.01 \mathrm{~K} .{ }^{5}$ In addition, each species is assumed to be either lipophilic, in which case it is capable of taking part in simulated reactions (type 1 or lipophilic) or it is lipophobic in which case it is extruded from the liposome and we assume it has a concentration of zero (type 0 of lipophobic). In most experiments there is a probability on 0.8 of a species being lipophilic. A third type, species extruded from the liposome but capable of taking part in surface reactions, is completely ignored for now. Type 0 molecules are not a part of the set of molecules inherited by offspring. Thus, the production of a lipophobic product can act to make a reaction effectively irreversible, even though chemically the equilibrium may not have been biased towards production of products.

The initial chemical reactor consists in most experiments of the following food set molecules, each provided at concentration $100 \mathrm{mM}$ : $\{a a b, a a a b, a a b b, b b b b, a a a a b$, $a a a b b, a a b b b\}$. All food set molecules are given a $G_{f}=1 \mathrm{~kJ}$.

\footnotetext{
${ }^{5}$ Note from the outset that there is no concept of bond energy or functional groups, i.e. the structure of a molecule does not deterministically define its free energy of formation. Instead, $G_{f}$ is assigned to species randomly, within conservation constraints. Neither is the activation energy of pathways between species assigned from assumptions about reaction mechanisms that can occur to interconvert strings. This would determine the kinetics, but here it is assumed that $k_{b}=0.01$ and $k_{f}=0.01 \mathrm{~K}$. Further elaborations on this technique of applying artificial selection to systems undergoing chemical variation by avalanches will make the structure of the chemistry more realistic.
}

The growth set is defined as the following set of molecules: $\{a b b, a b b b, a b b b b, a b b b b b, a b b b b b b, a b b b b b b b, a b b b b b b b b$, $a b b b b b b b b b\}$. No molecules of the growth set are present at initialization. The energy of growth set molecules differs. Those comprising the light absorbing reaction have energies defined as follows: $G_{a b b}=0.1 \mathrm{~kJ} \mathrm{~mol}^{-1}$, $G_{a b b b}=0.01 \mathrm{~kJ} \mathrm{~mol}^{-1}, G_{b a}=5 \mathrm{~kJ} \mathrm{~mol}^{-1}$. Thus, light is assumed to contribute apx $5 \mathrm{~kJ}$ of energy per light unit, and is present at a 'concentration' of 2 , and is treated effectively as a non-depleting species that undergoes a tri-molecular reaction with two $a b b$ molecules. The other members of the set have increasing $G_{f}$ with length, i.e. $G_{a b b b b}=1 \mathrm{~kJ} \mathrm{~mol}^{-1}, G_{a b b b b b}=2 \mathrm{~kJ} \mathrm{~mol}^{-1}, G_{a b b b b b b}=$ $3 \mathrm{~kJ} \mathrm{~mol}^{-1}$, etc. In some experiments an additional set of food molecules was included: $\{c c d, c c c d, c c d d, d d d d, c c c c d$, $c c c d d, c c d d d, c d d d d\}$. Note that no $c$ or $d$ atoms are included in molecules making up the growth set.

\subsection{Generation of avalanches}

In line 3 of the Algorithm 1 a chemical avalanche is generated as follows.

\section{Algorithm 2. Generate Avalanche}

0 : for $N=0$ to num_low_propensity_reactions.

1: Choose two existing species $(r 1, r 2)$ to react in a low propensity reaction $(k f \sim 0, k b \sim 0)$ to produce two potential products $p 1$ and $p 2$.

2: Generate the free energies of $p 1$ and $p 2$ (if they don't already exist) so that the following relation holds, $G_{p 1}+G_{p 2}+$ heat $=G_{r 1}+G_{r 2}$, by portioning energies according to a uniform random distribution, heat being positive.

3: if it is not possible to satisfy this relation, e.g. because $G_{p 1}>G_{r 1}+G_{r 2}$, then the reaction is not permitted, and no new products are created.

4: else if the condition that the reaction is spontaneous can be satisfied, create novel products at low concentration (e.g. $\left.10^{-7} \mathrm{M}\right)$ and increment newSpec $++/ /$ Store the number of novel species produced.

5: $k_{f}=k_{b}=0$. //Novel products come to existence at low concentration and are not produced, nor decay.

\section{6: end if}

7: end for

8: while newSpec $!=1$ do

9: $\quad$ for $i=0$ to newSpec

10: $\quad$ for $j=0$ to number of species currently existing

11: $\quad$ if $\operatorname{rand}()<$ prob_high_flux x $1 /$ (species[i].length $)^{2}$

12: $\quad$ tempNewSpecies = output of Algorithm 3

//Make high flux reaction with new

species[i] and a random species

13: $\quad$ end if

14: end for

15: end for

16: newSpec $=$ tempNewSpecies, tempNewSpecies $=0$.

17: end while 
Species produced in step 4: arise in a reaction which has effectively zero forward and backward rates, i.e. $k_{f}=k_{b}=0$, i.e. they are very rarely produced. For each new species produced at low concentration, one must check whether it reacts with other species. Line 11 describes our assumption that the longer the novel species the less likely it is to react with other species. If the condition on line 11 is passed, Algorithm 3 is carried out to generate a potential high flux reaction involving the novel species.

Algorithm 3. Calculate a high flux reaction

1: Choose species to react with rare species $i$ as follows.

2: for $j=0$ to num species

3: score $+=1 /(\text { length }[j] \text {-length }[i])^{2} \times(\text { length }[j])^{2}$

4: end for

5: Use roulette wheel selection to find species $\mathrm{j}$ biased by the above scores.

6: Once $r 1=i$ and $r 2$ have been chosen, generate random $p 1$ and $p 2$ products based on a bimolecular rearrangement of $r 1$ and $r 2$. This reaction can be biased in various ways, e.g. let the probability of a catalytic reaction, i.e. where $r 1=p 1$, or $r 2=p 2$, be related linearly to the proportion of ' $b$ ' atoms in $r 1$ or $r 2$. etc. Many such structure specific probabilistic rules may be applied.

7: Check that $G_{p 1}+G_{p 2}+$ heat $=G_{r 1}+G_{r 2}$ can be satisfied, and only if it can, store this new high flux reaction, and set $k_{f}=\operatorname{rand}(C) \times 0.01 \mathrm{~K}$, and

8: if $p 1$ OR $p 2$ already $/ / \mathrm{C}=100$ or 1000

exist, $k_{b}=0$

9: else

//This is to ensure that the

$\boldsymbol{k}_{\boldsymbol{b}}=\operatorname{rand}(C) \times 0.01$. exploration of the adjacent possible is correct

10: Return the number of new species produced legitimately, i.e. 0,1 or 2 .

\subsection{The definition of fitness}

Fitness is defined as the integral over the time of the trial of the product of [species[i]] $\times$ length of Species $i$, where $i$ is a molecule in the growth set. The second term introduces a biomass effect in which longer species contribute proportionally more to liposome growth. This is approximated by sampling concentrations at intervals of 1000 time steps. All trials are of fixed duration. The bolus of food molecules is allowed to deplete if the chemical avalanche has produced species that react with the bolus, or if material has been inherited that reacts with the bolus. This replicates the effect of a potential microfluidic experiment in which a single liposome is isolated in a small compartment containing a bolus of food molecules, and its size measured after a fixed duration. In many of the trials, fitness is assessed not on the first offspring produced after a chemical avalanche, but on the 3rd post-avalanche offspring. This is to increase the probability that any fitness benefit due to an avalanche is heritable, rather than

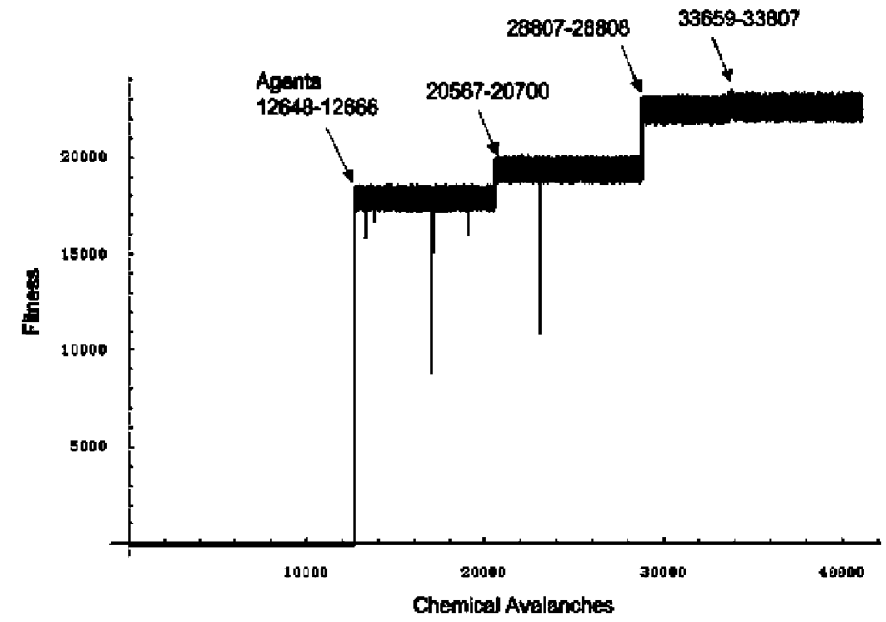

Fig. 1. Fitness of offspring produced over one evolutionary run. Four distinct punctuations are observed.

beneficial only to the offspring in which the avalanche occurs.

\section{Results}

Metabolic networks were evolved under various conditions. Representative samples are presented in Supplementary Material, showing one-step, two-step and three-step autocatalytic cycles that were evolved. The analysis will concentrate on one evolutionary run in which we found 4 distinct adaptations occurring by 4 chemical avalanches. ${ }^{6}$ Fig. 1 shows the fitness of the agents in the hill climbing algorithm over the course of the experiment. Initial fitness is zero since no $a b b$ or $a b b b$ exists. Therefore there is effectively random search for an avalanche that is capable of producing one or more members of the food set. The first adaptive avalanche is the 12648th. Following this, the frequency of adaptive avalanches is increased for the next three punctuations. Each punctuation consists of a relatively rapid succession of offspring that have higher fitness than the parent that started the punctuation. This may be because the original avalanche takes many generations to fully manifest its effects. After a punctuation, the offspring subjected to further chemical avalanches never return to the original fitness of zero. Note that there is a distinct population of harmful avalanches that reduce the fitness below the mean fitness of offspring produced after punctuation. These avalanches are those that produce an autocatalyst with a harmful effect on the liposome. Most avalanches do not produce an autocatalyst, and the fitness of such offspring contribute most to the mean fitness of post-punctuation offspring. Note that there are no

\footnotetext{
${ }^{6}$ The duration of each generation was set at 10,000 time-steps of size 0.0001 . This is reduced from the standard generation time of 100,000 timesteps used in most other runs, since it was found that autocatalytic adaptations typically occurred rapidly near the beginning of a generation, followed by a long period of steady-state (see supplementary material). Each run takes approx 12-24 h on an Apple Mac 2 GHz G5 duel processor computer.
} 
a

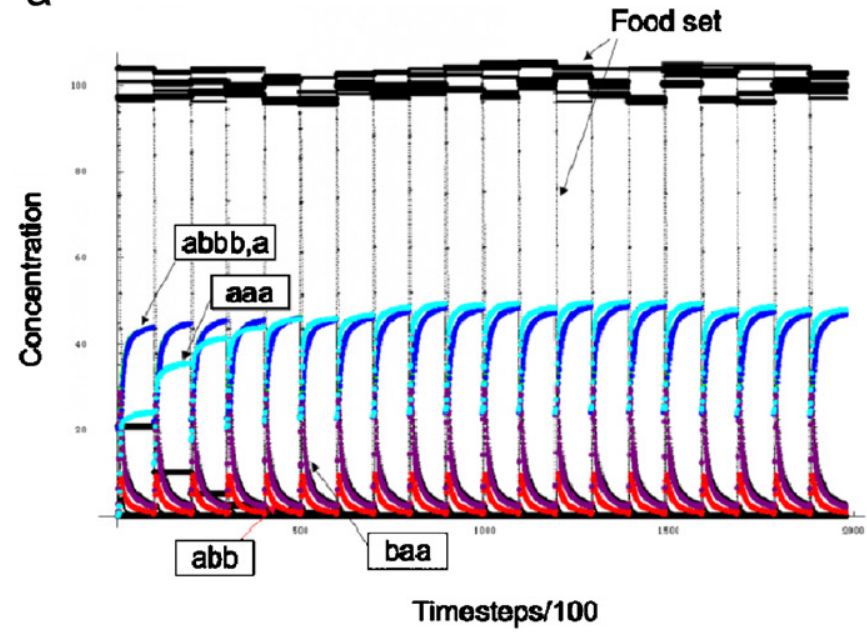

C

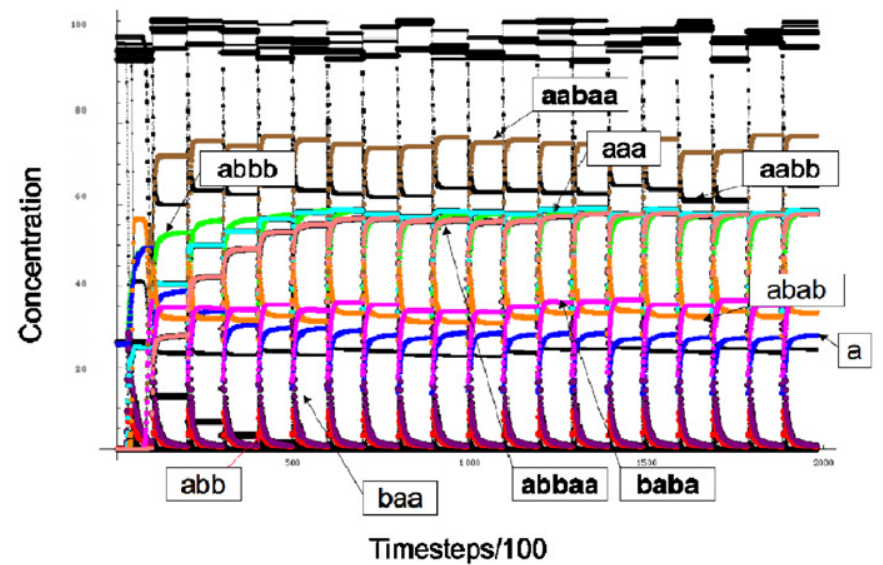

b

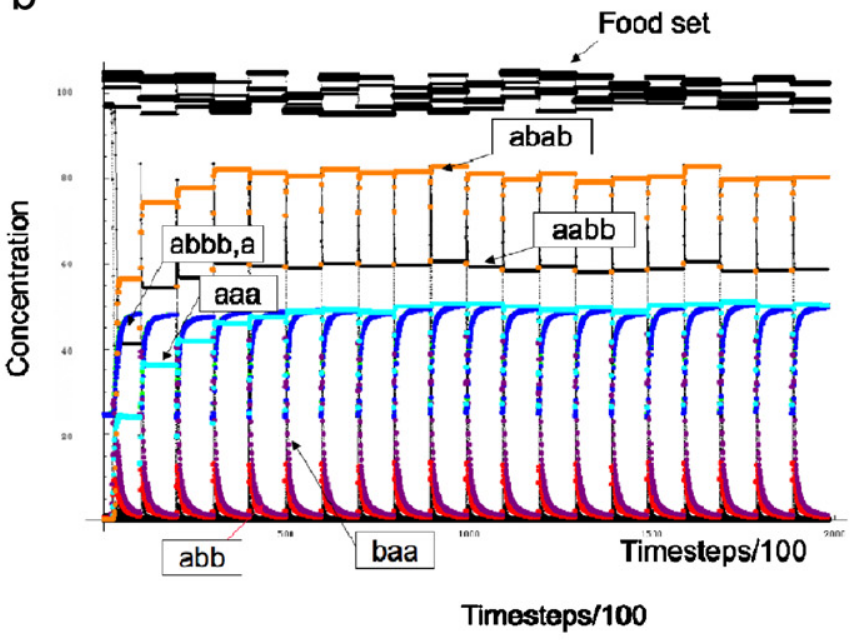

d

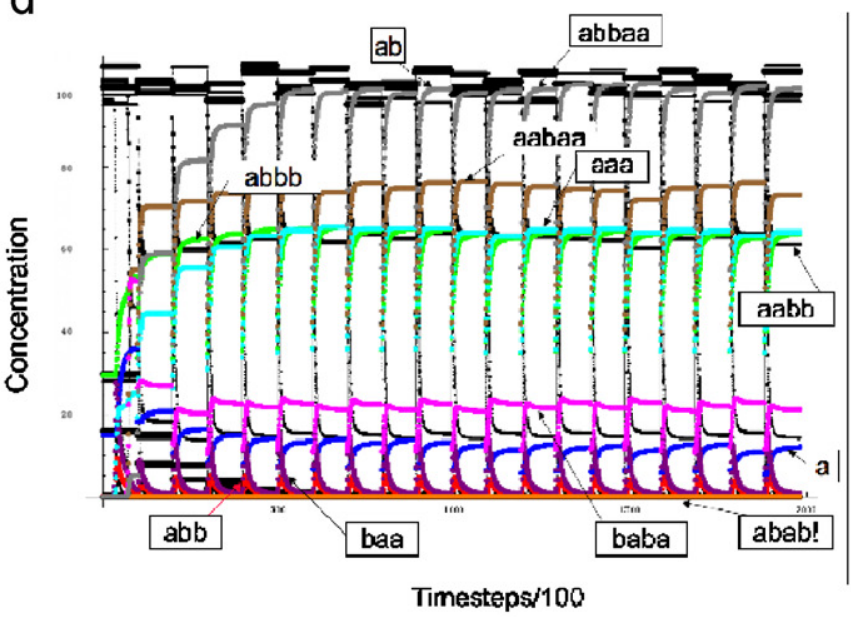

Fig. 2. Each graph shows the behaviour of an agent during 20 replication events in the absence of avalanches. Each generation lasts 10,000 time-steps. At each replication event, the lipophilic molecule concentration is halved and passed to the offspring, and the food bolus re-introduced at $100 \mathrm{mM} \pm 10 \mathrm{mM}$ per food molecule. Top Left (a): Agent 12,648. Top right (b): Agent 20,567. Bottom left (c): Agent 28,807. Bottom right (d): Agent 33,659.

outlying low fitness offspring after generation 28807 , which may be due to the absence of autocatalyst production. Each punctuation is followed by a rapid succession of offspring fitter than its parent. This is due to either the adaptive chemical avalanche requiring several generations to fully establish itself, or due to noise in the initial concentration of food set molecules provided.

The behaviour of the agents, one from each of the four fitness levels, is shown in Fig. 2. Note that with successive agents there is an increasingly complex concentration profile, i.e. more (non-food-set) species are maintained at high concentrations, that would be impossible without some form of 'memory' capable of withstanding dilution at each generation. For example between agent 12,648 and $20,567 a b a b$ has obtained the capacity to survive generational dilution. Between agent 20,567 and 28,807, three more species obtain high concentrations, aabaa, abbaa and baba. However, between agent 28,807 and 33,659 (admittedly a small fitness increase), we see a loss of species, not a gain, i.e. the concentration of $a b a b$ has become negligible.
Table 1

Properties of the 4 agents representative of each fitness plateau in Fig. 1

\begin{tabular}{lll}
\hline Agent I.D. & Num. species & Num. reactions \\
\hline 12648 & 61 & 47 \\
20567 & 99 & 63 \\
28807 & 122 & 76 \\
33659 & 147 & 89 \\
\hline
\end{tabular}

Since there is elitism in the hill-climbing algorithm, each agent is fitter than the previous agent. All agents gain fitness from the concentration of $a b b$ (red line) and $a b b b$ (green line). See how the height of $a b b b$ increases with each successive agent. Also note that $a b b b$ is initially at the same concentration as $a$, but that in the last two agents, $a b b b$ and $a$, have different concentration profiles, $a$ decreasing in concentration, and $a b b b$ increasing in concentration. No more complex growth set molecule is produced, e.g. $a b b b b$, $a b b b b b$ are completely absent. 


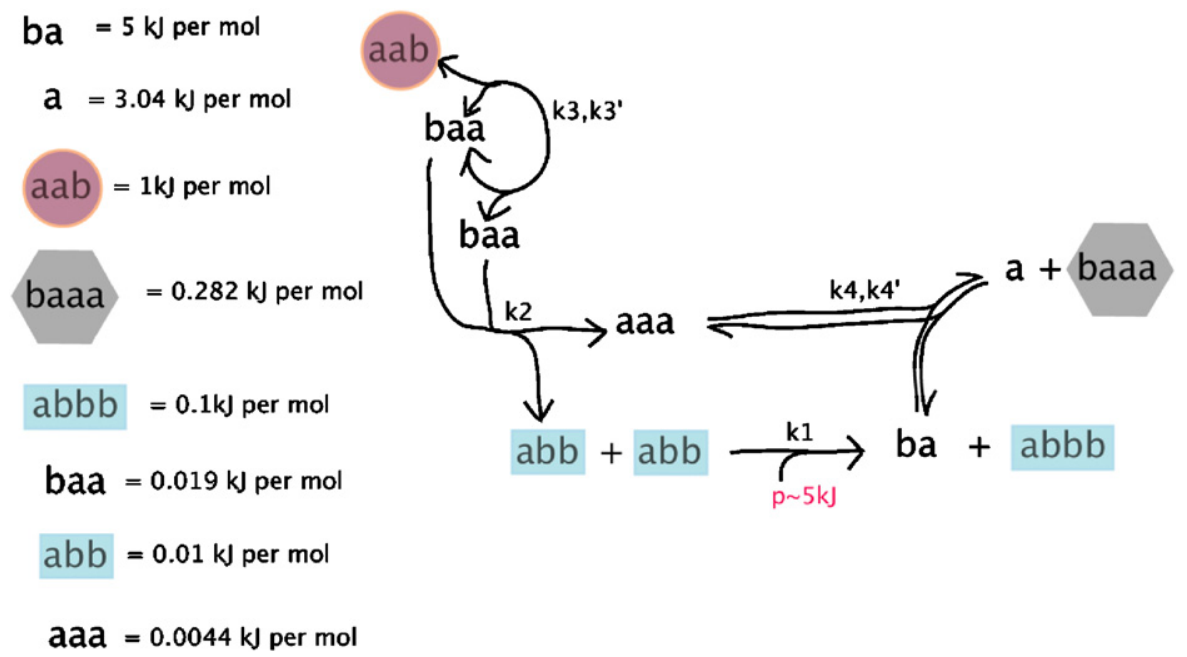

Fig. 3. The core autocatalytic network of agent 12,648. Red circle $=$ food molecule, blue rectangle $=$ growth molecule, grey hexagon $=$ lipophobic. All other reactions are not shown. $a a b$ is the only food molecule utilized. $b a a$ is the autocatalyst in one step. Two $b a a$ molecules are used to produce $a b b$ and $a a a$. $a b b$ then utilizes the existing light absorbing reaction to produce $b a$ and $a b b b$. $b a$ reacts with $a a a$ to produce $a+b a a a$. Since $b a a a$ is lipophobic, it leaves the liposome and would have driven this reaction in the forward direction, so encouraging the production of $a b b b$ from $a b b$. However, since the light absorbing reaction is irreversible, this extrusion reaction has no effect here. The $k$ values represent the catalytic factors, i.e. rand ${ }^{\bigcirc}$ in Algorithm 3 . These must be applied to the expression in Algorithm 3 to obtain the actual rates. $k_{1}=1.0, k_{2}=54.2136 k_{3}=66.3937, k_{3}^{\prime}=66.3937 . k_{4}=k_{4}^{\prime}=99.16$.

Each successive adaptive agent is increasingly complex. It has more species and more reactions, due to the accumulation of chemical avalanches. Table 1 shows the number of species and reactions that constitute the space of the chemical network utilized by accumulated chemical avalanches. Note that most of these species will be present at very low concentration due to the fact that most will not have been autocatalytically produced, and would have been present initially at very low concentration following an avalanche. Correspondingly, most reactions will experience very low flux.

It is possible, by hand, to identify the core components of the networks that are responsible for the behaviours shown in Fig. 2. Fig. 3 shows this analysis for agent 12,648. The core reaction network responsible for supra-basal fitness is due to the autocatalyst baa that is capable of using food molecule $a a b$. $b a a$ at the same time reacts with itself to produce $a b b+a a a . a b b$ is then transformed by the external energy absorbing reaction that was defined at the outset, to produce $b a+a b b b$. $a a a$ reacts with $b a$ producing $a+b a a a$, baaa being extruded from the liposome.

Fig. 4 shows how agent 20,567 is an improvement upon agent 12,648. A new single step autocatalytic constituent, $a b a b$, has been discovered by avalanche, along with a crosscatalysis of the direct production of an existing reaction intermediate (and autocatalyst!) baa, from a food molecule, $a a b$. We see in Fig. 2 the high concentration of $a b a b$ that is maintained by autocatalysis, and the lower concentration of $a a b b$ that is a consequence of this.

Fig. 5 shows how agent 28,807 has further improved upon agent 20,567. Another autocatalyst $b a b a$ has been produced which reacts with food molecule $a a b$ to produce $a b b b$ and $a a a$. Note that both $b a b a$ and $a b a b$ compete for the same food molecule $a a b b$.

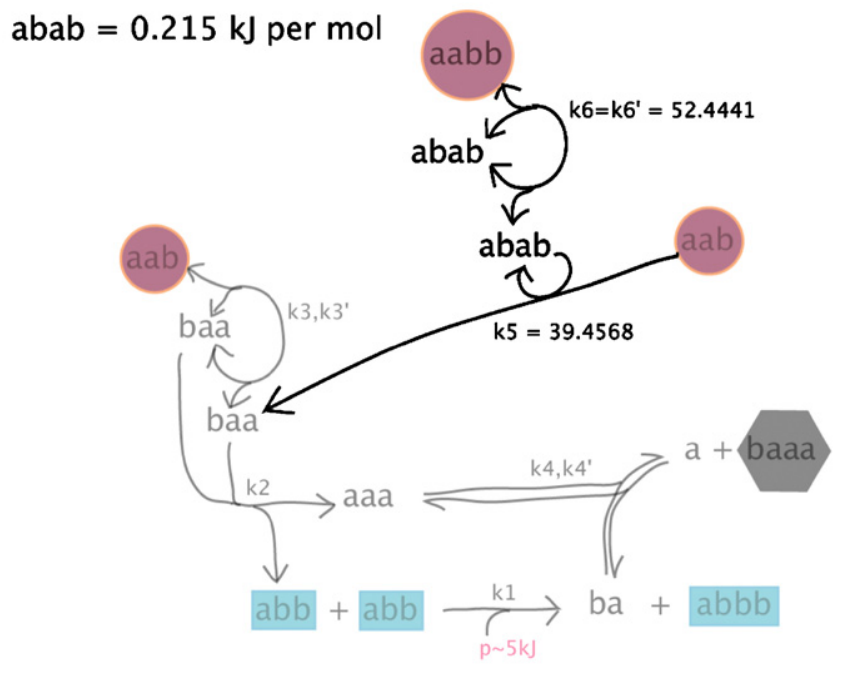

Fig. 4. The adaptation of agent 20,567 is a new autocatalytic particle with cross-catalytic effects to convert a food molecule into an existing reaction intermediate. The new reactions are shown in bold, and the old ones of agent 12,648 more faintly. There are two important new reactions evolved in agent 20,567. The first is a one step autocatalysis of $a b a b$ and the next is a catalysis by $a b a b$ of the conversion of food molecule $a a b$ into $b a a$, which then enters the same pathway as used by agent 12,648 to produce $a b b$ and $a b b b$.

Finally, it is important and remarkable that agent 33,659 although having experienced a new avalanche that produces no more obvious autocatalysts, has a greater fitness than agent 28,807 above. Why is this? It seems that the only difference between agent 33,659 and agent 28,807 is that the autocatalyst $a b a b$ and its dependent reactions have been lost, probably due to competition between $b a b a$ and $a b a b$ for food molecule $a a b b$. It just so happens that in 


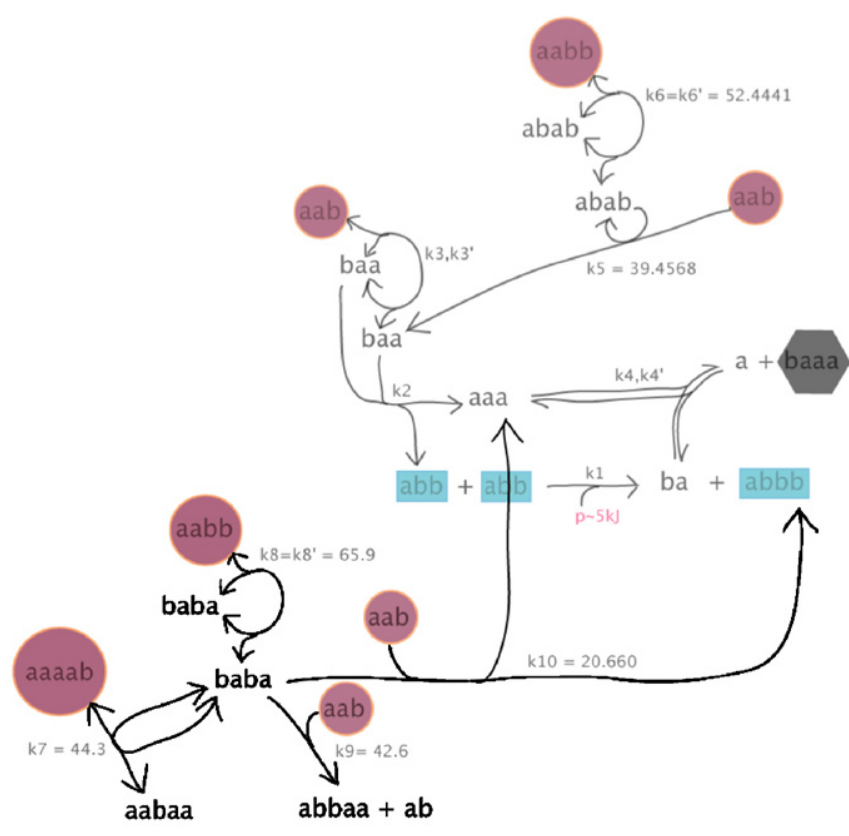

Fig. 5. Agent 28,807 exhibits another adaptation due to the discovery by avalanche of yet another autocatalyst $b a b a$ capable of replicating by using $a a b b$, and of producing $a b b b$ by reacting with $a a b . G_{b a b a}=0.0175 \mathrm{~kJ} \mathrm{~mol}^{-1}$. $G_{a a b a a}=0.556 \mathrm{~kJ} \mathrm{~mol}^{-1} . G_{a b b a a}=0.0044 \mathrm{~kJ} \mathrm{~mol}^{-1}, G_{a b}=0.292 \mathrm{~kJ} \mathrm{~mol}^{-1}$. It is evident that the reactions $a a a a b+b a b a \rightarrow a a b a a+b a b a$, and $b a b a+a a b \rightarrow$ $a b b a a+a b$, are probably harmful side-reactions that happen to have occurred in the same avalanche.

the absence of $a b a b, b a b a$ is actually able to produce $a b b b$ at a greater rate than before, so increasing fitness. The concentration changes between 28807 and 33659 in Fig. 2 are consistent with the above interpretation.

\section{Discussion}

\subsection{Autocatalysis is necessary for memory in the face of iterated dilution}

Chemical evolution by natural selection at the level of liposomes varying by chemical avalanches produces multiple autocatalytic cycles organized in various ways to produce the growth set. Several points are evident from the models.

Firstly, the theoretical argument of Wachtershauser is confirmed in this model (Wachtershauser, 1992). All the evolutionary runs (see Supplementary Material) demonstrate that selection at the level of the liposome results in survival of liposomes in which underlying molecular replicators came into existence. Natural selection acting at the level of the higher-order unit, the liposome, has stabilized novel lower-order units of selection. Why? Autocatalytic cycles are to be found in pre-enzymatic metabolism since only by autocatalysis is it possible to sustain a novel high flux reaction between generations, i.e. autocatalytic molecular replicators are the sole basis of memory. This type of autocatalysis should be called engram autocatalysis (Lashley,1950), to distinguish it from growth autocatalysis.
Growth autocatalysis refers to the autocatalytic production of any growth molecule, e.g. $a b b b$, which is defined as increasing the growth of the liposome, and so is a property at the level of the higher order unit.

Secondly, a shorter molecule of fewer atom types is more likely to produce itself in a random rearrangement reaction than is a longer molecule with more atom types, therefore the former have a greater probability of being autocatalytic constituents in the current model. This is critically dependent on the structural assumptions of the model, in real chemistry, an equivalence class of longer molecules may be capable of higher probability autocatalysis than some other equivalence class of shorter molecules. Further elaborations to our model of artificial chemistry are necessary to embody these more realistic structural influences, which in reality we conclude must be essential in continued adaptations. Importantly, only one, two, or three step autocatalytic cycles were evolved. This supports G.A.M. King's notion of a symbiotic process in which independently evolved small autocatalytic cycles come together displaying by-product mutualism (King, 1976). We have not experimented with amalgamation of liposomes, but such variation would occur naturally, and is a subject for further study.

Thirdly, independent autocatalysts can be sustained within the same liposome, especially if they utilize different food molecules, e.g. $b a b a$ uses $a a b b$, and $b a a$ uses $a a b$ as food and confer fitness to the liposome. There will be material constraints on the number of autocatalysts that can be maintained if these autocatalysts must compete for the same food molecule, as demonstrated in the final agent 33,659. In this case the autocatalyst with the lowest 'food threshold' will survive. Thus, in early systems we expect the original engram autocatalysts to have distinct food molecules.

Fourthly, autocatalysts can produce liposome level fitness by either catalytic reactions (e.g. $a b a b$ ), or by being consumed in reactions, that produce the growth set (e.g. baba). Both mechanisms are observed in the above experiment.

Fifthly, the build-up of lipophilic side-reactants such as aabaa produced reversibly in an OR-reaction tapping an autocatalytic cycle can actually be beneficial to the liposome since this reduces the net efflux of $b a b a$ from the cycle. Conversely, the removal of a hydrophilic product can shift the equilibrium towards the products, e.g. potentially baaa. In the absence of large differences in chemical potential, random production of lipophilic and hydrophilic species can be a 'poor man's irreversibility' by physical rather than chemical potentials.

Sixthly, the autocatalytic cycles are coupled not by a cross-catalytic requirement for each other's production, but by the fact that they contribute to the same intermediary metabolism that produces the growth set. Of-course, it did happen in our run that baa became secondarily the product of a new autocatalytic cycle, but its production never became entirely dependent on that novel cycle, in fact the novel cycle was lost. Parasitic autocatalysts, i.e. autocatalysts that use another autocatalyst as 
their food molecule, can be more easily sustained when the food molecules are of higher free energy.

Seventhly, the probability of producing an autocatalytic cycle in an avalanche increases as the diversity of molecules in the food set increases [results not shown]. This is because there are more chances that a multiple reaction autocatalytic cycle will be successfully driven at each step, without the requirement for an extra source of internal recycling at each step (note this applies most importantly to bimolecular rearrangements).

Eigthly, the frequency of autocatalyst production decreases with each chemical avalanche, see Fig. 7 (middle).

\subsection{Energy dissipation tends to increase with increasing fitness}

Since this model has conservation of energy, we can ask; is there a relationship between fitness and energy dissipation within a lifetime, or between fitness and the energetic inheritance transferred to offspring during the division event? Fig. 6 (middle) shows that the lower bound of energy dissipation increases with increasing fitness, but that high energy dissipation does not inevitably result in high fitness, for example, offspring with fitness zero show high rates of energy dissipation, but this is due to reactions that do not produce the growth set and so do not increase fitness. Fig. 6, (bottom) shows the energy dissipation over the course of 20 division events in agent 33,659. Obviously, most of the energy dissipation occurs at the beginning of a lifetime since this is when the food bolus is supplied.

Why does energy dissipation increase with increasing fitness? There are two quite distinct causes of energy dissipation, the first is the production of growth set molecules from food molecules, and the second is the maintenance of engram autocatalysts, required to sustain the network capable of producing growth set molecules. The total energy dissipation per generation must inevitably increase as more growth set molecules are produced. Since fitness is here defined as proportional to the mass of the growth set molecules produced after a fixed time period, this component of energy dissipation must inevitably increase with increasing fitness. Of-course, in reality, fitness is not directly related to the mass of material that constitutes the organization of the individual, because units of selection can evolve to divide at a lower mass. Also, organisms may evolve to make the free energy of the growth set greater than that of the food set, in which case external energy sources other than food will have to be utilized, and there will be a net gain of energy, rather than a net loss. However, in the current model, the high free energy growth set molecules were not discovered. This is partly because their production in a spontaneous reaction occurring in a chemical avalanche is less likely than the production of a low free energy molecule (see Fig. 8, bottom right), but also because they are longer.

The second component of energy dissipation is due to engram autocatalysis. Unlike the component of energy
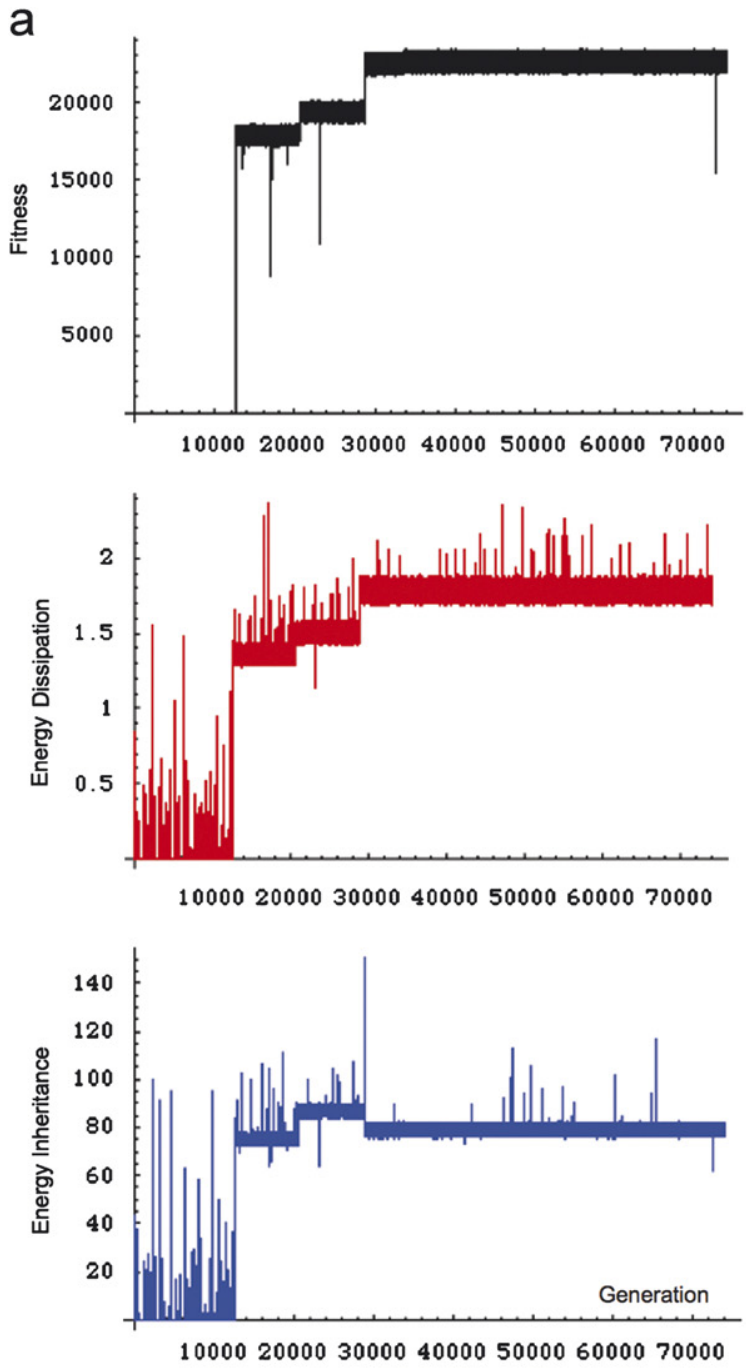

b

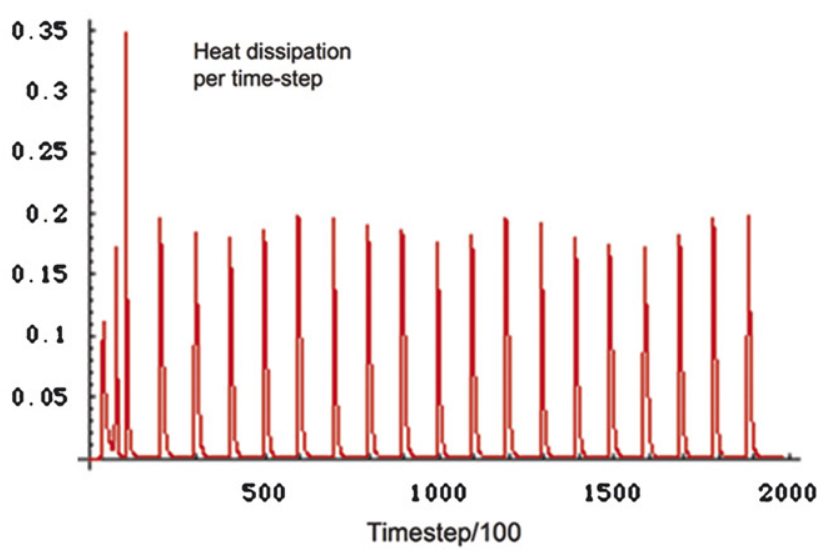

Fig. 6. (a) Top: Fitness of agents during the evolutionary run of the hillclimbing algorithm. Top Middle: Total energy dissipation per generation, measured as the sum over all reactions, of flux through reaction $i \times$ free energy change in reaction i. Bottom Middle: Energy inherited, measured as the sum of concentration $x$ free energy of all lipophilic species at the beginning of a generation, not including the food set. (b) Right Bottom: Energy dissipation per time step over 20 divisions of agent 33,659 . No distinction between heat and entropy components of energy has been made in this model. 
dissipation due to production of the growth set, this component does not necessarily have to increase with increasing fitness in this model, because an engram autocatalyst of higher free energy may maintain a reaction by cross-catalysis, better than another engram autocatalyst with a lower free energy of formation. Alternatively, an organism may have higher fitness with fewer well-placed autocatalysts than another organism that uses a greater number of energetically costly but poorly placed autocatalysts.

Despite these reasons why an increase of energy dissipation is not inevitable, it is nevertheless observed. The main reason being that more low energy molecules are produced in chemical avalanches than high-energy molecules, since reactions in chemical avalanches must occur spontaneously. In addition, there is no selection pressure for more efficient liposomes, i.e. for liposomes that produce the growth set with the minimal quantity of food molecules. A fitness function that explicitly rewarded efficiency of growth set production may be expected to produce liposomes with lower energy dissipation per generation. Such a fitness function would simulate conditions of competition for limited food molecules. However, where variation is capable of producing organisms capable of utilizing novel energy sources, we expect the total energy dissipation of the biosphere to increase, even though individual organisms would be selected that could utilize the novel energy source more efficiently.

Finally, the total free energy of chemicals transmitted to offspring does not necessarily increase with fitness (see Fig. 6, 3rd down).

\subsection{Properties of avalanches relate to the frequency of adaptations}

Fig. 7 reveals why no adaptation arises after generation 34,000 . The number of autocatalytically growing molecules produced in an avalanche decreases with each adaptation, as does the frequency of producing at least one autocatalyst per avalanche. This is because the mean length of species produced in an avalanche increases with each adaptation. Avalanches become less effective at producing adaptations as they begin to produce more divergent reactions with fewer cycles.

Fig. 8 shows 4 histograms of avalanche properties: number of reactions, number of species, mean species length, and species free energy. Note the log-normal type distribution of reaction sizes and species numbers per avalanche, and the tendency for most species to have low free energies of formation, with only a few species having high free energies of formation.

\subsection{A stochastic population model of chemical evolution by natural selection}

The previous model used a hill-climbing algorithm that contained elitism. In reality there is no elitism. It may be
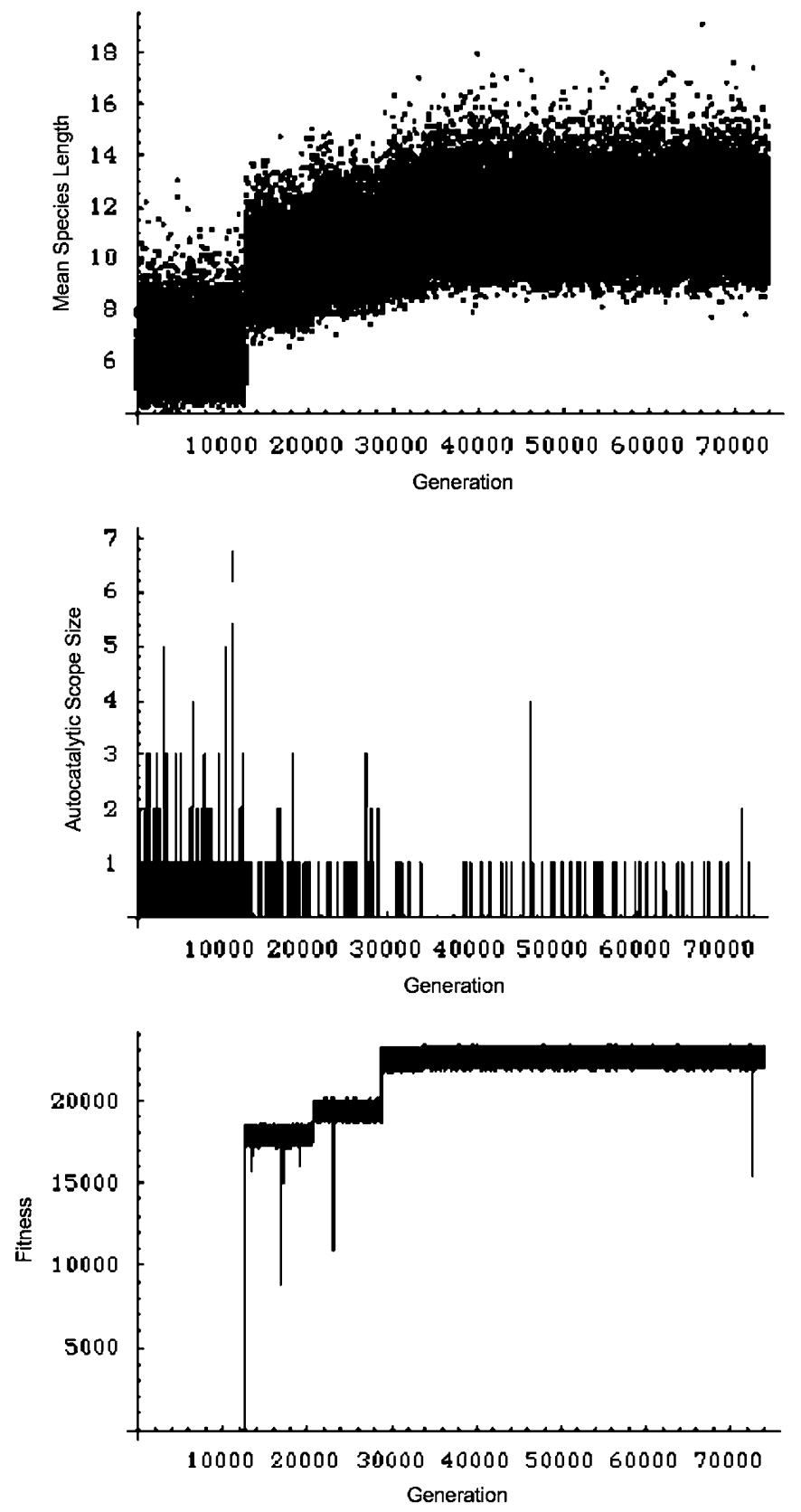

Fig. 7. Top: Mean species length produced during an avalanche, over the course of apx 70,000 generations. There is an increase in the mean length of species produced. Middle: The extent of influence of autocatalytic reactions upon the chemical network, and the frequency of autocatalytic reactions, can be visualized by plotting the number of novel species whose concentration reaches at least $0.0001 \mathrm{mM}$ within one generation. Note that all novel species are initialized at a concentration of $10^{-7} \mathrm{mM}$. Bottom: Fitness as shown in Fig. 1.

the case that if too many low fitness offspring are produced per fit offspring, in a finite population, that Muller's ratchet will operate (Muller, 1932). This model tests the conditions in which chemical evolution by natural selection can occur in finite populations when we assume that most avalanches are harmful, that autocatalysts can be lost, and that population sizes may be relatively small. Fig. 9 gives a rough outline of the chemical experiment. The chemist 

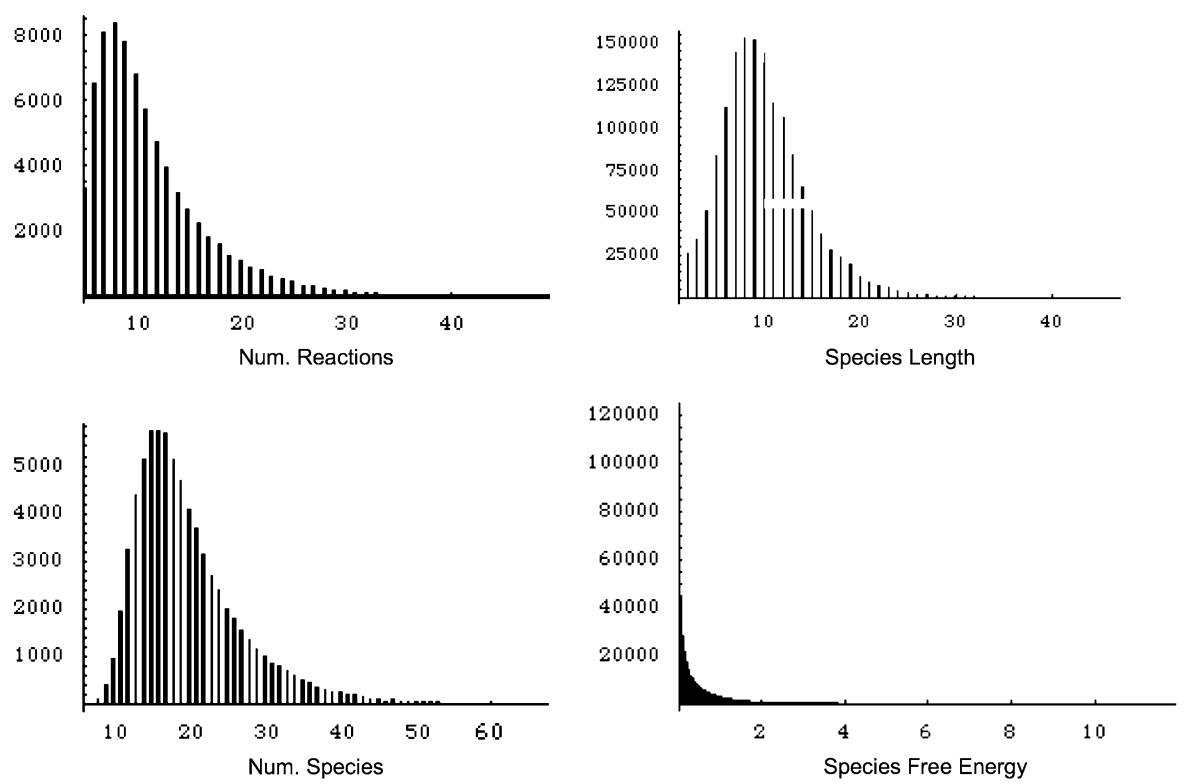

Fig. 8. Histograms of avalanche properties. Top left: Histogram of frequency vs. number of reactions produced in avalanches, over the entire course of evolution in Fig. 1. Top right: Histogram of species lengths produced in each avalanche. Bottom left: Histogram of number of species produced in each avalanche. Bottom right: Histogram of species free energies produced in each avalanche.

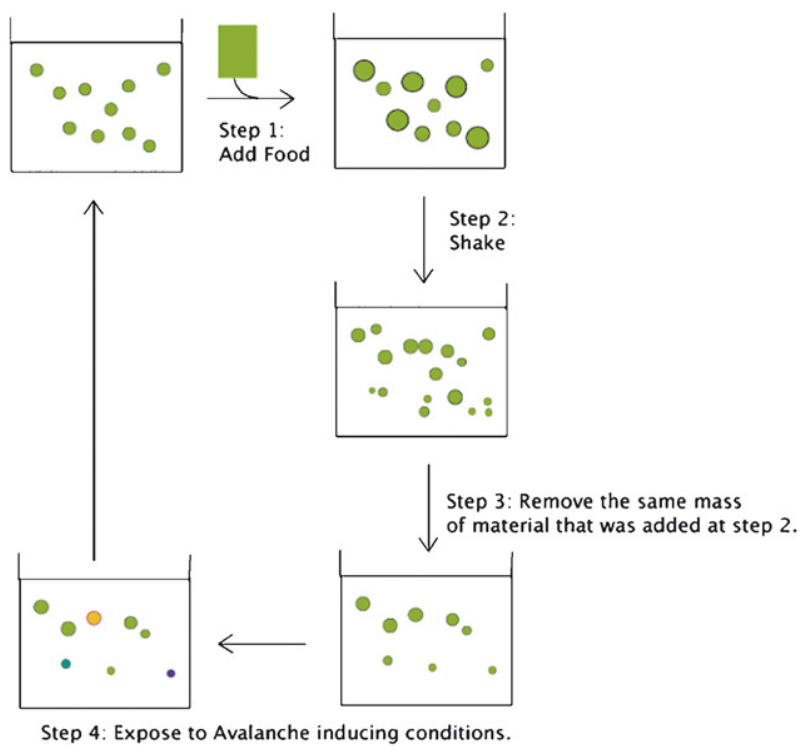

Fig. 9. Start with an initial set of $N$ liposomes, each of size $S$. Each liposome is described by variables under evolutionary control. $G=$ capacity to grow when food is added, $D=$ capacity to divide when reactor is vigorously shaken. Avalanches occur with some probability $Q$, and have some probability $P$ of producing an autocatalyst and of altering $G$ and $D$, according to some distribution. Most avalanches will reduce $G$ and $D$, and may even kill the liposome, i.e. make it dissipate, with some probability $K$. We assume that engram autocatalysis has a failure rate, $F$, i.e. that there is some probability of a beneficial autocatalyst being lost, with a corresponding loss of the component of influence by that autocatalyst on $G$ and $D$. We ask under what conditions a chemist would expect to observe the accumulation of beneficial autocatalysts in the reactor, i.e. those autocatalysts that maximize the fitness of its liposome.

starts with $N=1000$ liposomes of volume $S=1$ in a reactor and adds a known volume $M=1000$ of material that can form liposomes. We assume that a proportion
$W=0.1$ of material goes to producing novel liposomes, whilst the rest contributes to the growth of existing ones. The efficiency with which a liposome absorbs material is an evolvable liposome property, $G$. Once the system has reached a steady state in the size distribution of liposomes after addition of food, the reactor is agitated so that the liposomes divide, approximately symmetrically, and perhaps for multiple 'generations' in one shaking event. The probability of a liposome splitting upon shaking is an evolvable liposome property, $D$. A volume $M$ of randomly chosen liposomes is removed from the reactor, i.e. liposome volume is kept approximately constant between generations by random harvesting of liposomes. This exerts selective pressure. The reactor state is then changed to increase the probability of a chemical avalanche occurring per liposome to a small value, $Q=0.01$ per unit volume. If an avalanche occurs, there is a probability $P=0.01$ of that avalanche producing an autocatalyst, and if an autocatalyst is produced there is a certain distribution of effects on $G$, and $D$ in that liposome, the effects being highly skewed to the detrimental, i.e. most autocatalysts will reduce $G$ or $D$ and will with a probability $K=0.9$, destroy the liposome completely, e.g. by causing it to dissipate. The cycle iterates as another food bolus is added.

How does $G$ and $D$ influence growth and division? The new size of a liposome, $x$, after the growth phase is given by the equation...

$S_{t+1}^{x}=S_{t}^{x}+\frac{M(1-W) G_{x}}{\sum_{i=0}^{i=N} G_{i}}$,

i.e. the new size, $S_{t+1}$, is the previous size, $S_{t}$, plus the proportion of food volume available for existing liposomes, $M(1-W)$, times the contribution that goes to this liposome, determined by $G_{x}$ of liposome $x$ divided by the sum of $G$ 
over all liposomes. A novel set of liposomes is produced with the remaining food volume $M W$, at the original size, which is set at 1 for convenience. Division is modelled as follows. The shaking period consists of for example $\mathrm{H}$ 'shakes'. At each shake (of which there are 10 per round) a liposome divides if

$\operatorname{rand}()<D^{x}\left(S^{x}\right)^{2}$ where $\operatorname{rand}()$ is a random number between 0 and 1 , and $S$ is the size of the liposome $x$. How do we choose the distribution of autocatalyst effects on $G$ and $D$ ? $G$ is initialized at 1 , and $D$ is initialized at 0.1 . There is a probability $h$ that an autocatalyst reduces $G$ or $D$ by a uniform random number between zero and one, multiplied by its current value, and a probability $g=1-h$ that an autocatalyst increases $G$ or $D$ by a uniform random

a
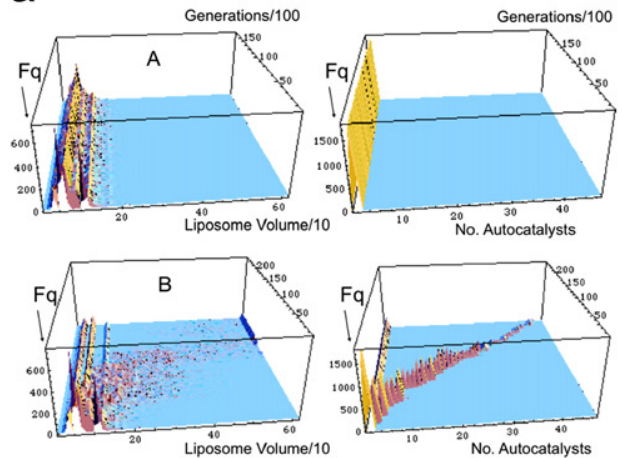

d

b
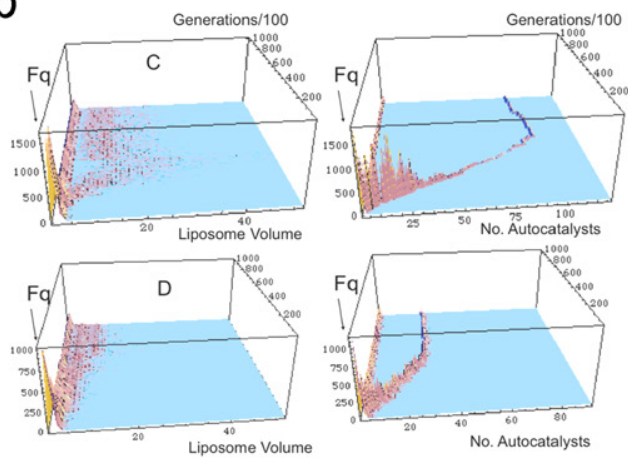

C
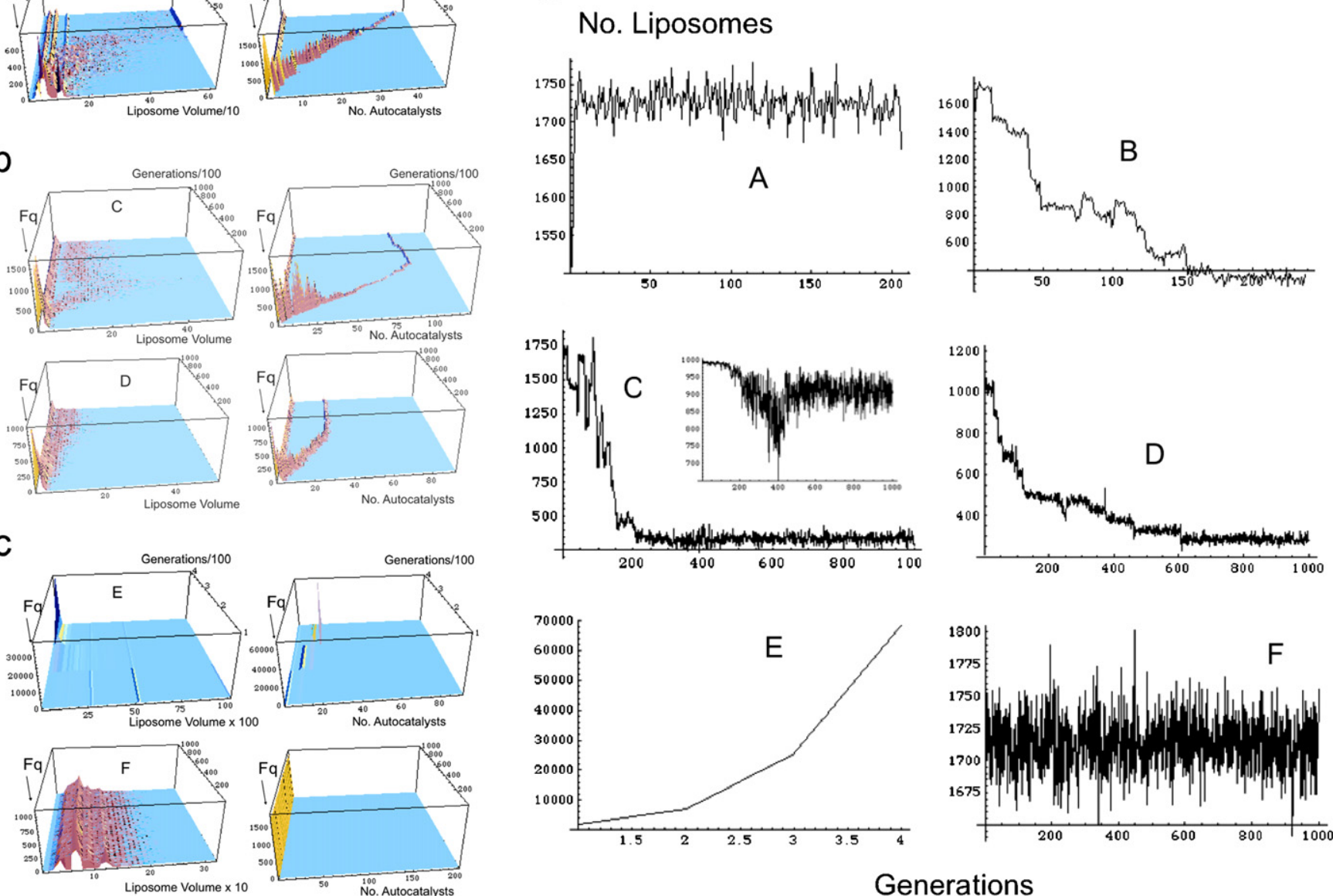

Fig. 10. Simulations of variants of the experiment shown in Fig. 9. Generations refers to one iteration of the experimental loop in Fig. 9 not to real liposome generations; the later may be more numerous because each generation consists of 10 'shakes', with a division possible in each 'shake'. a(A): Control run with no chemical avalanches. Most liposomes are small volume (see d(A)). a(B): High probability avalanches $(Q=P=0.1), g=1 / 100$. Autocatalysts steadily accumulate, with $G$ increasing, and $D$ decreasing (not shown), resulting in larger and fewer liposomes $\mathrm{d}(\mathrm{B})$. b(C): Very high probability avalanches $(Q=P=1.0)$ and $g=1 / 100$. Eventually there is no further increase in autocatalyst number, and although there is a brief peak in liposome size, eventually the size decreases and reaches a steady state $($ see $d(C))$. The inset in $d(C)$ shows the average liposome volume in this run. $b(D)$ : Very high probability avalanches $(Q=P=1.0)$ and $g=1 / 10000$, i.e. a larger harmful 'mutation load'. The autocatalyst number does not increase to as high a value as before an evolutionary steady-state is reached. The number of liposomes decreases d(D). c(E): High probability avalanches $(Q=P=0.1)$, and $g=1 / 100$ but with $g=9999 / 10000 . D$ and $G$ decrease, producing much smaller and more numerous liposomes (d(E). Only 400 generations of the simulation could be run in reasonable time. Autocatalysts accumulate (approximately 15 within 400 generations). c $(\mathrm{F}):(Q=P=1.0)$ and $g=1 / 100$. However, the effect (positive or negative) of an autocatalyst is only $0.001 \times$ the magnitude of the effect on $G$ or $D$ compared to the previous runs. No autocatalysts can be established, and the size distribution of liposomes is the basal one as in the first experiment (note different length scale) $d(F)$. 
number between 1 and 2 times its current value. Typically, $g=1 / 100$, i.e. the probability of a beneficial autocatalyst is one in 100 autocatalysts produced. There is a probability $K=0.9$ that an avalanche results in the complete loss of the liposome. Furthermore, at division, there is a probability $F=0.000001$, per autocatalyst per generation that the autocatalyst will be lost due to segregation instability.

We seek sufficient conditions for the accumulation of adaptations by chemical avalanches in this system. Six conditions are examined in Fig. 10. The first is a control run in the absence of chemical avalanches; the second run is with high probability avalanches $(Q=P=0.1)$ and $g=1 / 100$; the third run is with very high probability avalanches $(Q=P=1.0)$ and $g=1 / 100$; the forth run is with very high probability avalanches $(Q=P=1.0)$ and $g=1 / 10000$, i.e. a larger harmful 'mutation load'; the fifth run is with high probability avalanches $(Q=P=0.1)$ and $g=9999 / 10000$, i.e. it is assumed that most autocatalysts have the opposite effect, i.e. to increase $G$ and $D$, rather than to decrease them; the final run is again with high probability avalanches $(Q=P=0.1)$, and $g=1 / 100$ but this time with only $0.001 \times$ the magnitude of the effect on $G$ or $D$ compared to the previous runs.

The main points of note are that autocatalysts can be accumulated under a wide range of conditions, providing the magnitude of their effect on fitness is sufficiently high given the population sizes concerned. Only in case $d(F)$ above where the effect on fitness of autocatalysis was very small did autocatalysts not accumulate. The high proportion of 'harmful' autocatalysts to 'beneficial' autocatalysts is of little concern. However, the main limitation for the chemist may be the rate at which novel autocatalysts can be made to occur in the laboratory by chemical avalanches. In the computer runs, a high probability (per generation, per liposome) of an avalanche producing an autocatalyst was assumed. If avalanche rates are low, more generations are required to accumulate adaptations.

\section{Conclusions}

We hypothesized a geophysical natural selection machine capable of sustaining chemical evolution by natural selection in which heterotrophically formed liposomes replicate by external agitation at a base rate and experience chemical avalanches which affect their growth rate. We modelled an artificial selection experiment using a hillclimbing algorithm in which a liposome is selected for maximizing the production of a subset of chemicals that defines its growth set, from a food set supplied as a bolus in each generation. We found that autocatalytic cycles evolved in the liposomes because they were necessary for the maintenance of chemical reaction networks that were beneficial at the liposome level. The segregation into liposome individuals allows armful avalanches to be isolated to just one compartment. This is especially important in primitive evolutionary systems in which most chemical avalanches are harmful. Fontana and Kauffman's models do not demand a population of individuals because they ignore the problem of harmful side-reactions that would destroy the entire system unless they could be isolated.

The idealization of two distinct kinetic time scales (fast and slow) is a convenience for the model. In fact, chemical avalanches would be expected to occur according to a more complex distribution of periods. The rate of chemical avalanches would have to be sufficiently low to allow a liposome experiencing a successful avalanche to reach fixation before its progeny were wiped out by the much more frequent harmful avalanches. The capacity for variation by chemical avalanches depends on there being requisite variety, but this is an assumption that probably cannot be avoided in any successful constructive dynamical system (Ashby, 1956).

Chemists are encouraged to pursue experiments involving heterotrophically produced phase separated chemical units as units of selection. A note of caution; if the effect of autocatalysts is small, then very large population sizes are required. If the frequency of chemical avalanches is too low, then some means of accelerating this process must be devised, e.g. the exposure of liposomes to high pressure between generations, or the capacity to reduce generation times by automation.

\section{Acknowledgements}

Thanks to Eörs Szathmáry for crucially important inspiration and guidance. We wish to thank the European 6th Framework Project ESIGNET for financial support in the development of stochastic models of chemical networks.

\section{Appendix A. Supplementary materials}

Supplementary data associated with this article can be found in the online version at doi:10.1016/j.jtbi.2007.01.028.

\section{References}

Ashby, W.R., 1956. An Introduction to Cybernetics. Part Two: Variety. London, England, Methuen.

Bagley, R., Farmer, J., 1991. Spontaneous emergence of a metabolism. In: Langton, C.G., et al. (Eds.), Artificial Life II. SFI Studies in Science of Complexity. Addison-Wiley.

Bahadur, K., 1954. Nature 173, 1141-1142.

Bahadur, K., Ranganayaki, S., 1983. Synthesis of Jeewanu, the protocells and study of their functional properties namely the ability to split water in sunlight and to fix molecular nitrogen and carbox dioxide. Lecture. 7th ISSOL Conference, Mainz.

Bedau, M., McCaskill, J.S., Packard, N.H., Rasmussen, S., Adami, C., Green, D.G., Ikegami, T., Kaneko, K., Ray, T.S., 2000. Open problems in artificial life. Artificial Life 6, 363-376.

Decker, P., Schweer, H., Pohlmann, R., 1982. Bioids. Identification of formose sugars, presumable porebiotic metabolites, using capillary gas chromorography/gas chromotography-mass spectrosopy of $n$-butoxime trifluroacetates on ov-225. J. Chrom. 255, 281-291. 
Dewer, R.C., 2005. Maximum entropy production and the fluctuation theorem. J. Phys. A: Math. Gen. 38, L371-L381.

Dittrich, P., Speroni, d.F.P., 2007. Chemical Organization Theory; Towards a theory of constructive dynamical systems. Bull. Math. Biology. in press.

Dyson, F., 1985. Origins of Life. Cambridge University Press.

Eigen, M., Schuster, P., 1977. The Hypercycle. A principle of natural selforganization. A: Emergence of the Hypercycle. Naturwissenshaffen 64, 541-565.

Eigen, M., 1971. Self-organization of matter and evolution of biological macromolecules. Naturwissenschaften 58 (10), 465-523.

Farmer, J.D., Kauffman, S.E., Packard, N.D., 1986. Autocatalytic replication of polymers. Physica D 22, 50.

Folsome, C.E., 1979. The Origin of Life: A Warm Little Pond. WH Freeman and Company.

Fontana, W., Buss, L., 1994. 'The arrival of the fittest': toward a theory of biological organization. Bull. Math. Biol. 56, 1-64.

Fox, S.W., Dose, K., 1977. Molecular Evolution and the Origin of Life. Marcel Decker Inc.

Ganti, T., 2003a. The Principles of Life, Oxford University Press, New York. [Eors Szathmary, James Griesemer, (Eds.), Based on Tibor Ganti. The Principle of Life, Gondolat, Budapest, 1971 (In Hungarian)].

Ganti, T., 2003b. Chemoton Theory, the Theory of Living Systems, Vol. 2. Kluwer Publishers.

Haldane, J.B.S., 1954. The origins of life. New Biol. 16, 12-27.

Hogeweg, P., Takeuchi, N., 2003. Multilevel selection in models of prebiotic evolution: compartments and spatial self-organization. Orig. Life. Evol. Biosph. 33 (4-5), 375-403.

Jain, S., Krishna, S., 1998. Autocatalytic sets and the growth of complexity in an evolutionary model. Phys. Rev. Lett. 81, 5684-5687.

Kauffman, S.A., 1986. Autocatalytic sets of proteins. J. Theor. Biol. 119, $1-24$.

King, G.A.M., 1976. Symbiosis and the origin of life. Origins Life Evol. Biospheres 8, 39-53.

King, G.A.M., 1982. Recycling, reproduction, and life's origins. Biosystems $15,89-97$.

Kleidon, K., 2004. Beyond Gaia: thermodynamics of life and Earth system functioning. Climatic Change 66, 271-319.

Lashley, K., 1950. In search of the engram. Symp. Soc. Exp. Biol. 4, 454-482.

Lee, D.H., Severin, K., Yokobayashi, Y., Ghadiri, M.R., 1997. A selfreplicating peptide. Nature 390, 591 [Erratum (1998) Nature 394, 10].

McGregor, S., Fernando, C., 2005. Levels of description: A novel Approach to dynamical hierarchies. Artificial Life 11, 459-472.

Maturana, H., Verela, F., 1992. The Tree of Knowledge: the Biological Roots of Human Understanding. Shambhala.

Maynard-Smith, J., Szathmary, E., 1995. The Major Transitions in Evolution. Oxford University Press, Oxford, UK.
Maynard-Smith, J., 1986. The Problems of Biology. Oxford University Press, Oxford, UK.

Miller, S.L., 1953. A production of amino acids under possible primitive earth conditions. Science 117, 528.

Miller, S.L., Orgel, L., 1974. The Origins of Life on Earth. Prentice-Hall.

Morowitz, H.J., 1968. Energy Flow in Biology: Biological Organization as a Problem in Thermal Physics. Academic Press, New York, London.

Morowitz, H.J., 1971. An energetic approach to prebiological chemistry. In: Buvet, R., Ponnamperuma, C. (Eds.), Chemical Evolution and the Origin of Life. North-Holland Publishing company.

Morowitz, H.J., 1992. Beginnings of Cellular Life: Metabolism Recapitulates Biogenesis. Yale University Press.

Mossel, E., Steel, M., 2005. Random biochemical networks: the probability of self-sustaining autocatalysis. J. Theor. Biol. 233 (3), 327-336.

Muller, H.J., 1932. Some Genetic Aspects of Sex. Am. Naturalist 66, $118-138$.

Oparin, A.I., 1924. Proiskhozhdenie Zhizni. Moskovskji Rabochij, Moscow.

Oparin, A.I., 1938. The Origin of Life. (1953 translation, Dover Publishers).

Oparin, A.I., 1964. The Chemical Origin of Life Trans. Ann. Synge. Charles C Thomas.

Orgel, L., 2000. Self-organizing biochemical cycles. PNAS 97 (23), 12503-12507.

Rich, E., Knight, K., 1991. Artificial Intelligence. McGraw-Hill, New York.

Ruiz-Mirazo, K., Pereto, J., Moreno, A., 2004. A universal definition of life: autonomy and open-ended evolution. Orig. Life. Evol. Biosph. 34 (3), 323-346.

Schuster, P., 2000. Taming combinatorial explosion. PNAS 9 (14), 7678-7680.

Segre, D., Ben-Eli, D., Lancet, D., 2000. Compositional genomes: prebiotic information transfer in mutually catalytic noncovalent assemblies. Proc. Natl. Acad. Sci. USA 97 (8), 4112-4117.

Segre, D., Ben-Eli, D., Deamer, D.W., Lancet, D., 2001. The lipid world. Origins LifeEvol. Biospheres 31 (1-2), 119-145.

Szathmary, E., 1995. A classification of replicators and lambda-calculus models of biological organization. Proc. Roy. Soc.: Biol. Sci. 260, 279-286.

Szathmary, E., 2000. The evolution of replicators. Phil. Trans. Roy. Soc. Lond. B 355, 1669-1676.

Szathmary, E., Santos, M., Fernando, C.T., 2005. Evolutionary Potential and Requirements for Minimal Protocells. Topics in Current Chemistry. Springr, Berlin, Heidelberg.

Wachtershauser, G., 1992. Groundworks for an evolutionary biochemistry: The iron-sulphur world. Prog. Biophys. Molec. Biol. 58, 85-201.

Weber, A.L., 2005. Growth of organic microspherules in sugar-ammonia reactions. Origins Life Evol. Biosphere 35, 523-536. 